\title{
BRONQUIOLITIS OBLITERANTE SECUNDARIA A SINDROME DE STEVENS-JOHNSON. PRESENTACION DE 2 CASOS Y REVISION DE LA LITERATURA
}

\author{
OBLITERANS BRONCHIOLITIS SECONDARY TO STEVENS-JOHNSON SYNDROME. \\ CASE REPORTS
}

\author{
Dr. Julio Maggiolo M. ${ }^{1}$, Dra Lilian Rubilar 0. ${ }^{1}$ \\ 1 Pediatra Especialista en Enfermedades Respiratorias. Unidad Broncopulmonar. Hospital Exequiel González Cortés.
}

ABSTRACT

Stevens-Johnson syndrome corresponds to a hypersensitivity reaction produced by various etiologies, for example exposure to drugs, microbial agents, or by an idiopathic cause. It is marked by an acute vesicular-bullous eruption, which affects the skin and mucous membranes, with systemic manifestations of variable severity, and it may present a fatal evolution.

Stevens-Johnson syndrome can occasionally present chronic pulmonary complications, such as bronchiolitis obliterans; however, other etiologies are more frequent in our environment, for example severe pneumonia due to adenovirus. Our objective is to present two cases of bronchiolitis obliterans post Stevens-Johnson syndrome and to make a literature review.

Keywords: Stevens-Johnson syndrome, skin, mucous membrane, bronchiolitis obliterans.

RESUMEN

El síndrome de Stevens-Johnson corresponde a una respuesta de hipersensibilidad producida por diversas etiologías, que incluyen exposición a drogas, agentes microbianos o idiopática. Se manifiesta por una erupción vesículo-bulosa aguda, que afecta la piel y las mucosas, con manifestaciones sistémicas de severidad variable, pudiendo presentar una evolución fatal.

El síndrome de Stevens-Johnson puede presentar ocasionalmente complicaciones pulmonares crónicas, como bronquiolitis obliterante, siendo en nuestro medio más frecuente otras etiologías, como la observada luego de una neumonía grave por adenovirus. El objetivo es presentar dos casos de bronquiolitis obliterante post síndrome de Stevens-Johnson y hacer una revisión de la literatura. Keywords: Palabras clave: sindrome Stevens Johnson, piel, mucosas, bronquiolitis obliterante.

\section{INTRODUCCIÓN}

El síndrome de Stevens-Johnson (SSJ) o eritema multiforme mayor, corresponde a una enfermedad poco frecuente, inflamatoria aguda, caracterizada por una erupción vesículo-bulosa aguda, que afecta la piel y las mucosas, con manifestaciones sistémicas de severidad variable (1). Representa una reacción de hipersensibilidad a diversos agentes etiológicos, con mayor frecuencia frente a la exposición a drogas de uso habitual como antibióticos, anticonvulsivantes y antiinflamatorios no esteroidales.

Las complicaciones agudas del aparato respiratorio que han sido descritas en el SSJ incluyen edema laríngeo, epiglotitis, bronquiolitis, neumonía, neumotórax, neumomediastino. Sin embargo las complicaciones pulmonares crónicas, como bronquiolitis obliterante (B0) y enfermedad pulmonar intersticial crónica (EPIC) (2-6), se observan menos frecuentemente, siendo en nuestro medio más habitual otras etiologías, como la observada luego de una neumonía grave por Adenovirus (7).

Nuestro objetivo es presentar dos casos de BO secundaria a un SSJ y hacer una revisión de la literatura.

\footnotetext{
Correspondencia:

Dr Julio Maggiolo

Hospital Dr Exequiel González Cortés

Gran Avenida José Miguel Carrera 3300

San Miguel, Santiago

Correo electrónico: maggiolojulio@gmail.com
}

\section{CASOS CLÍNICOS}

\section{Caso clínico 1}

Paciente varón, a los 9 años de edad en relación a una infección respiratoria alta febril recibe diclofenaco sódico intramuscular (IM). A las pocas horas se evidencia una erupción maculo-papular en cara, tronco y extremidades, se indica betametasona IM. El paciente evoluciona con decaimiento, exantema vesículo-buloso, lesiones en "tiro al blanco", comprometiendo palmas y plantas, enantema vesículoulceroso en boca y conjuntiva, además edema bipalpebral, tos y dificultad respiratoria leve, requiere oxígeno, antihistamínicos y corticoides sistémicos, presentando buena evolución, con lenta recuperación de las lesiones de piel y mucosas. Posteriormente presenta crisis bronquiales obstructivas mensuales y broncorrea persistente.

Derivado a nuestra unidad a los 12 años, al examen físico destaca murmullo pulmonar globalmente disminuido, roncus y sibilancias bilaterales, sin lesiones cutáneas. Evaluación oftalmológica normal.

Se sospecha BO post SSJ. La radiografía (Rx) de tórax muestra hiperinsuflación severa, imágenes intersticiales y peribronquiales difusas (Figura 1). La tomografía computada (TC) de tórax evidencia patrón en mosaico, bronquiectasias cilíndricas y quísticas, múltiples impactaciones mucosas (Figura 2).

La espirometría basal y curva flujo-volumen muestran un patrón ventilatorio obstructivo avanzado, con cambios significativos, sin normalización de valores post B2, curva 
espiratoria cóncava (Figura 3). No responde al test esteroidal. Los volúmenes pulmonares demuestran capacidad pulmonar total normal con atrapamiento aéreo, post $\beta 2$ discreta disminución del atrapamiento aéreo. Test de marcha de 6 minutos (TM6') mostró un compromiso importante utilizando los valores de referencia de Li y cols (Figura 4). Saturometría nocturna normal.

Actualmente el paciente tiene 16 años de edad, ha presentado reagudizaciones infecciosas frecuentes, con limitación de la actividad física.

Figura 1. Radiografía de tórax antero-posterior.

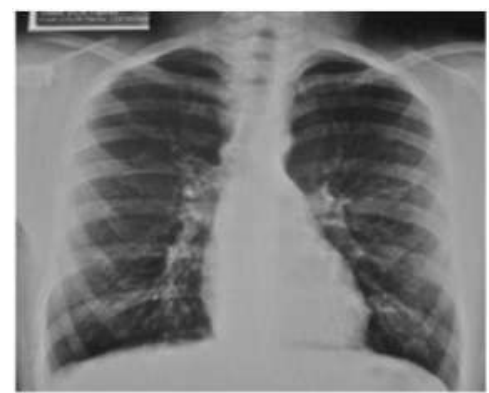

Hiperinsuflación severa, imágenes peribronquiales e intersticiales difusas.

Figura 2. Tomografía computada de tórax.

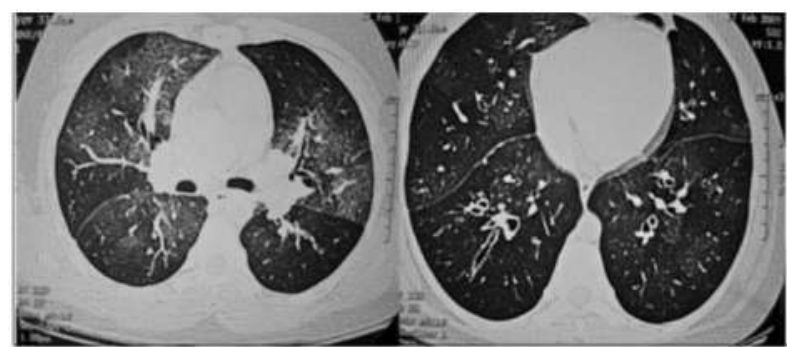

Patrón en mosaico bilateral, bronquiectasias cilíndricas y quísticas, múltiples impactaciones mucosas.

Figura 3. Espirometría basal y post broncodilatador, curva flujo/volumen.

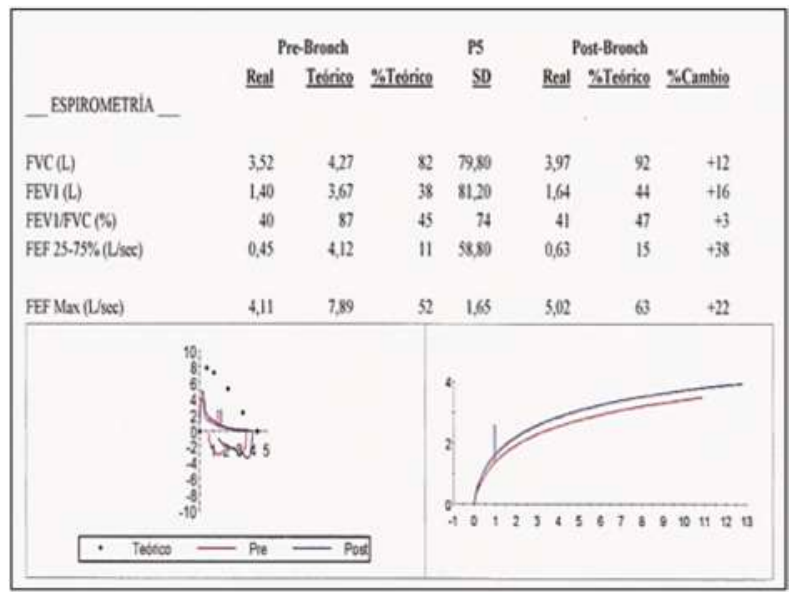

Patrón ventilatorio obstructivo avanzado, post B2 cambios significativos, sin normalización de valores. Curva espiratoria cóncava.
Figura 4. Prueba de marcha 6 minutos.

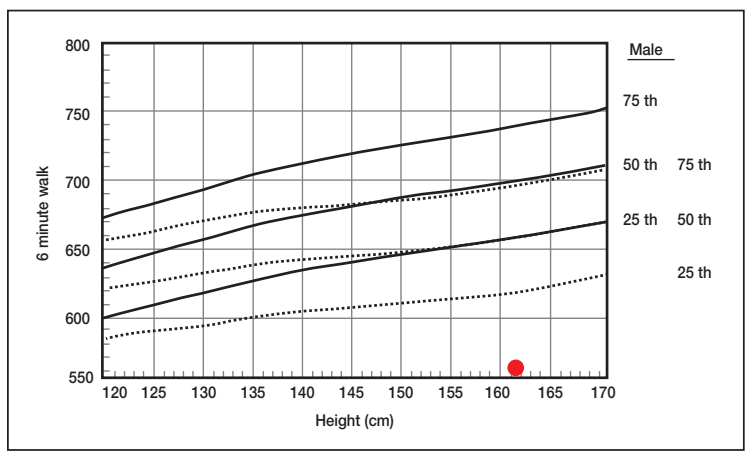

El círculo rojo muestra el resultado del paciente.

\section{Caso clínico 2}

Paciente mujer, con agenesia parcial del cuerpo calloso, a los 5 años se diagnostica epilepsia. Inicia carbamazepina, al quinto día comienza con lesiones eritematosas, máculopapulares, ampollares, en tronco, posteriormente se extienden al resto de la piel, comprometiendo mucosa oral y conjuntival, diagnosticándose SSJ. Presenta una neumonía bacteriana con insuficiencia respiratoria aguda, requiere ventilación mecánica invasiva (VMI) 11 días, diferentes esquemas antibióticos y hospitalización de 30 días.

Evoluciona satisfactoriamente del compromiso cutáneo-mucoso, presentando escasas cicatrices, pero persiste con tos productiva, motivo por el cual se deriva a nuestra unidad a los 7 años de edad.

Se sospecha B0 post SSJ, se indica fluticasona 500 ug/día/salmeterol 100 ug/día, se solicita TC tórax que muestra mosaico de perfusión, no colabora con exámenes de función pulmonar. Permanece relativamente estable durante 3 años.

Figura 5. Tomografía computada de tórax.



Hiperinsuflación, mosaico de perfusión, bronquiectasias en anillo lóbulo medio y língula e impactaciones mucosas.

A los 11 años de edad nuevamente presenta reagudizaciones infecciosas y obstructivas bronquiales frecuentes, en la última se prescribe amoxicilina/ácido clavulánico y luego ciprofloxacino, sin mejoría, se solicitan cultivos de hongos que resultan positivos para Aspergillus fumigatus, IgE total y específica con títulos elevados, una nueva TC tórax evidencia mosaico perfusión, bronquiectasias en anillo en lóbulo 
medio (LM) y língula (Figura 5), se diagnostica Aspergilosis broncopulmonar alérgica (ABPA) y se inicia tratamiento con bolos de metilprednisolona y voriconazol, presentando buena respuesta.

Actualmente la paciente tiene 13 años de edad, presenta 2 crisis infecciosas anuales.

\section{DISCUSIÓN}

Las complicaciones pulmonares agudas de SSJ tales como neumonía y bronquiolitis han sido bien documentadas. En cambio las complicaciones crónicas son infrecuentes, como la $\mathrm{B} 0$, la que habitualmente es severa y progresiva (2-6), menos frecuentemente ha sido descrita la EPIC.

La BO se caracteriza por ser una forma fibrosante de Ios bronquíolos, que conduce a un estrechamiento u obliteración completa de la vía aérea pequeña, lo que configura una enfermedad pulmonar obstructiva fija y crónica, producida luego de una injuria severa (7-10).

En pediatría la etiología más frecuente en nuestro medio es la que se produce luego de una infección severa de la vía aérea inferior (Adenovirus, Mycoplasma pneumoniae), en tanto el SSJ es una causa poco frecuente (7-10). En la Tabla 1 se enumeran las causas más importante de B0 en los niños (7-10).

Tabla 1. Etiología de bronquiolitis obliterante en pediatría.

\begin{tabular}{|c|c|}
\hline Infecciones & $\begin{array}{l}\text { Adenovirus tipo } 3,7 \text { y } 21 \\
\text { Influenza } \\
\text { Parainfluenza } \\
\text { Virus sincicial respiratorio } \\
\text { Sarampión } \\
\text { Varicela } \\
\text { Bordetella pertussis } \\
\text { Mycoplasma pneumoniae }\end{array}$ \\
\hline Trasplante & $\begin{array}{l}\text { Rechazo crónico del trasplante corazón-pulmón } \\
\text { Enfermedad injerto versus huésped asociada al } \\
\text { trasplante de médula ósea }\end{array}$ \\
\hline $\begin{array}{l}\text { Enfermedades del tejido } \\
\text { Conectivo }\end{array}$ & $\begin{array}{l}\text { Artritis Reumatoidea } \\
\text { Enfermedad de Sjogren } \\
\text { Lupus Eritematoso Sistémico }\end{array}$ \\
\hline Aspiración de gases & $\begin{array}{l}\mathrm{NO} 2 \\
\mathrm{NH} 3\end{array}$ \\
\hline Neumonitis por hipersensibilidad & $\begin{array}{l}\text { Antigenos de aves } \\
\text { Hongos }\end{array}$ \\
\hline Aspiración & $\begin{array}{l}\text { Reflujo gastroesofágico } \\
\text { Cuerpo extraño } \\
\text { Penicilamina } \\
\text { Cocaína }\end{array}$ \\
\hline Sindrome de Stevens-Johnson & $\begin{array}{l}\text { Idiopático } \\
\text { Inducido por drogas } \\
\text { Relacionado a infecciones }\end{array}$ \\
\hline
\end{tabular}

La patogénesis de las secuelas pulmonares crónicas en el SSJ no está del todo aclarada, los mecanismos que han sido propuestos son como resultado de la lesión primaria en el tracto respiratorio poducido por la noxa, secundaria a infección pulmonar y por último mecanismo inmune tipo III con depósitos de complejos inmunes, especialmente cuando están involucrados medicamentos, o como resultado de la combinación de ellos (8).

Por otro lado ha sido descrita la asociación de BO post
SSJ y afectación del tracto gastrointestinal, como esofagitis y posterior estenosis esofágica (11).

Algunos autores sin contar con la histopatología, han reportado el comienzo de $\mathrm{B} 0$ en el período de convalescencia, 5 a 10 meses luego de presentar SSJ (2). Ha sido descrito un paciente con falla respiratoria aguda (12), otros desarrollaron una $\mathrm{B} 0$ rápidamente progresiva, 15 días luego del comienzo del SSJ (13).

Tanto los niños descritos en la literatura y nuestros pacientes presentaron tos y dificultad respiratoria en la fase aguda, seguido por un período de escasa sintomatología con posterior empeoramiento, la aparición de signos y síntomas respiratorios crónicos ocurrió 3 a 4 meses después del inicio del SSJ.

A pesar de no contar con datos histopatológicos en nuestros pacientes, el diagnóstico de BO se estableció por la persistencia y progresión de los síntomas respiratorios, más el patrón radiológico y de función pulmonar.

La biopsia pulmonar es el gold standard en el diagnóstico de B0 $(14,15)$, sin embargo habitualmente no es practicada (8), bastando con TC de tórax $(16,17)$. Desde el punto de vista imagenológico tanto el patrón en mosaico, atelectasias y bronquiectasias, fueron hallazgos comunes en todos los casos $(16,17)$.

En cuanto a la función pulmonar se observó en un paciente alteración ventilatoria avanzada con cambios significativos, sin normalización de valores post beta2, test esteroidal no demostró reversibilidad.

La evolución a largo plazo de los pacientes reportados en la literatura presentaron mortalidad e importante morbilidad, esto último coincidente con nuestros pacientes, quienes han presentado sintomatología pulmonar persistente debida a reagudizaciones infecciosas y uno presentó ABPA (6-13).

El trasplante pulmonar debe ser considerado como última opción en casos severos y progresivos.

Los autores declaran no presentar conflicto de intereses.

\section{REFERENCIAS}

1. Stitt VJ Jr. Stevens-Johnson syndrome: a review of the literature. J Natl Med Assoc 1988; 80: 104-8.

2. Virant FS, Redding GJ, Novack AH. Multiple pulmonary complications in a patient with Stevens-Johnson syndrome. Clin Pediar (Phila) 1984; 23: 412-14.

3. Park H, Ko YB, Kwon H, Lim C. Bronchiolitis obliterans associated with Stevens-Johnson Syndrome: A case report. Yonsei Med J. 2015; 56: 578-81.

4. Yatsunami J, Nakanishi Y, Matsuki H, Wakamatsu, Takayama K, Kawasaki Ogino, Hashimoto S, Hara N. Chronic Bronchobronchiolitis Obliterans Associated with StevensJohnson Syndrome. Intern Med1995; 34: 772-5.

5. Basker M, Cherian T, Raghupathy P. Chronic Lung Disease Following Stevens-Johnson Syndrome. Indian Pediatr 1997; 34: 831-5.

6. Bakirtas A, Harmanci K, Toyran M, Razi C, Turktas I. Bronchiolitis Obliterans: A Rare Chronic Pulmonary 
Complication Associated with Stevens-Johnson Syndrome. Pediatr Dermatol 2007; 24: E22-E25.

7. Comisión multidisciplinaria para el estudio de la Bronquiolitis Obliterante. Consenso para el diagnóstico y cuidado de niños/adolescentes con Bronquiolitis Obliterante postinfecciosa. Neumol Pediatr 2009; 4: 1-52.

8. Moonnumakal SP, Fan LL. Bronchiolitis obliterans in children. Curr Opin Pediatr 2008; 20: 272-78.

9. Chiu CY, Wong KS, Huang YCh, Lin TY. Bronchiolitis obliterans in children: Clinical presentation, therapy and long-term follow-up. J Paediatr Child Health 2008; 44: 129-33.

10. Kurland G, Michelson P. Bronchiolitis Obliterans in Children (State of the Art). Pediatr Pulmonol 2005; 39: 193-208.

11. Edell DS, Davidson JJ, Muelenaer AA, Majure M. Unusual Manifestation of Stevens-Johnson Syndrome Involving the Respiratory and Gastrointestinal Tract. Pediatr 1992; 89: 429-32.

12. Schamberger MS, Goel J, Braddock SR, Parsons DS, Tobias JD. Stevens-Johnson syndrome and respiratory failure in a 9-year-old boy. Southern Med J 1997; 90: 755-7.

13. Tsunoda N, Iwanaga $T$, Saito $T$, et al. Rapidly progressive bronchiolitis obliterans associated Stevens-Johnson syndrome. Chest 1990; 98: 243-5.

14. Mauad T, Dolhnikoff $M$, and Sao Paulo Bronchiolitis Obliterans Study Group. Histology of Childhood Bronchiolitis Obliterans. Pediatr Pulmonol 2002; 33: 466-74.

15. Sugino K, Hebisawa A, Uekusa T, Hatanaka K, Abe H, Homma S. Bronchiolitis obliterans associated with Stevens-Johnson Syndrome: histopathological bronchial reconstruction of the whole lung and immunohistochemical study. Diagn Pathol 2013; 8: 134.

16. Padley SP, Adler BD, Hansell DM, Muller NL. Bronchiolitis obliterans: high resolution CT findings and correlation with pulmonary function test. Clin Radiol 1993; 47: 236-40.

17. Kim MJ, Lee KY. Bronchiolitis obliterans in children with Stevens-Johnson syndrome: follow-up with high resolution CT. Pediatr Radiol 1996; 26: 22-5. 